Recepción: 27 / 05 / 2018

\title{
Optimización de las condiciones de extracción y parámetros cromatográficos en la determinación de amigdalina en las almendras dulces y amargas
}

\section{Optimization of extraction conditions and chromatographic parameters in the determination of amygdalin in sweet and bitter almonds}

\author{
María M. Morales-Padilla ${ }^{\mathrm{I}}$ \\ Universidad Internacional Iberoamericana UNINI \\ mamopa2@alumni.upv.es \\ Jacqueline del Rocío Ávila-Jácome II \\ jacqueaj84@yahoo.es \\ Pascual Barrera-Alina III \\ Departamento de Tecnología de Alimentos, Universidad Politècnica de València \\ pascualbarrera@gmail.com
}

Correspondencia: $\underline{\text { mamopa2@alumni.upv.es }}$

I. Docente de la Universidad Internacional Iberoamericana UNINI, Ciudad de México, México.

II. Universidad Internacional Iberoamericana UNINI, Ciudad de México, México.

III. Departamento de Tecnología de Alimentos, Universidad Politécnica de Valencia. 


\section{Resumen}

La almendra es una drupa de semilla comestible, consumida y comercializada en su estado natural o como parte de diversos productos elaborados. Se distinguen dos clases de almendras: amargas y dulces. El sabor amargo se debe a la presencia de compuestos cianogénicos, como la amigdalina, que es un compuesto altamente toxico que influye directamente en la calidad sensorial y aceptabilidad del producto.

En este estudio se determinó el contenido de amigdalina en almendras mediante cromatografía líquida de alta resolución (HPLC), optimizando, tanto las condiciones de extracción, como las condiciones de separación de la amigdalina de las almendras, obteniéndose mejores resultados con una fase móvil compuesta por una mezcla de agua y acetonitrilo $(80: 20, \mathrm{v} / \mathrm{v})$ en elución isocrática con un caudal de $1 \mathrm{~mL} \cdot \mathrm{min} 1 \mathrm{y}$ un volumen de inyección de $20 \mu \mathrm{L}$. El método cromatográfico desarrollado dio lugar a repetibilidades inter-día con desviaciones estándar relativas (RSD) menores de 0,31 y $1,41 \%$ para tiempos de retención y áreas de pico, respectivamente. Además, se obtuvo un límite de detección (LOD) del $0,40 \mathrm{mg} \cdot \mathrm{L}-1$ y recuperaciones en todos los casos cercanas al $100 \%$. El contenido de amigdalina obtenido en almendras dulces varió en un rango de 0,67 a $348 \mathrm{mg} \cdot \mathrm{kg}-1$, en tanto que para las almendras amargas se detectó un contenido de amigdalina entre 14734 y $50412 \mathrm{mg} \cdot \mathrm{kg}-1$

Palabras claves: Amigdalina, Cianogénico, Cromatografía, Límite de detección, Tóxico. 
The almond is an edible seed, consumed and marketed in its natural state or as part of processed products. There are two kinds of almonds: bitter and sweet. The bitter taste is due to the presence of cyanogenic compounds, such as amygdalin, which is a highly toxic compound that directly influences the sensory quality and acceptability of the product.

In this study, the content of amygdalin in almonds was determined by high performance liquid chromatography (HPLC), the conditions of extraction and the separation conditions of the amygdalin in the almonds were optimized, methanol being selected as the solvent and a $24 \mathrm{~h}$ stirring time. The best results were obtained with a mobile phase composed of a mixture of water and acetonitrile $(80: 20, \mathrm{v} / \mathrm{v})$ in isocratic elution with a flow rate of $1 \mathrm{~mL}$ min-1 and a volume of injection of $20 \mu \mathrm{L}$. The chromatographic method developed gave rise to inter-day repeatability with relative standard deviations (RSDs) of less than 0.31 and $1.41 \%$ for retention times and peak areas, respectively. In addition, a detection limit (LOD) of $0.40 \mathrm{mg} \cdot \mathrm{L}-1$ and recoveries were obtained in all cases close to $100 \%$. The content of amygdalin obtained in sweet almonds ranged from 0.67 to 348 $\mathrm{mg} \cdot \mathrm{kg}-1$, whereas for bitter almonds the amygdalin content detected was between 14734 and 50412 $\mathrm{mg} \cdot \mathrm{kg}-1$.

Keywords: Amigdalin, Cyanogenic, Chromatography, Detection limit, Toxic. 
María M. Morales-Padilla; Jacqueline del Rocío Ávila-Jácome; Pascual Barrera-Alina

\section{Introducción.}

Las almendras dulces son un fruto comestible en su estado natural y muy utilizado como ingrediente principal en productos alimenticios manufacturados. El grano puede ser secado, tostado, frito, triturado o utilizado en pasta para fabricar diversos productos de panadería. Sin embargo, las almendras amargas también se utilizan, principalmente, en la producción de extractos de sabor, siendo previamente procesadas para eliminar las sustancias indeseables.

La presencia de almendras amargas en un producto elaborado con almendras dulces causa un sabor desagradable que influye directamente en la calidad y aceptabilidad del producto. Este sabor amargo se debe a la presencia de glucósidos cianogénicos, como la amigdalina y la prunasina. La amigdalina se encuentra en el núcleo de las almendras, mientras que la prunasina es un monoglucósido que se encuentra en raíces, hojas y granos de almendras inmaduras y que se convierte en amigdalina durante el proceso de maduración. El sabor amargo se presenta debido a la hidrólisis enzimática por la $\beta$ - glucosidasa que produce benzaldehídos, azúcares y cianuro de hidrógeno, proporcionando una barrera de defensa química contra los herbívoros, insectos y patógenos; sin embargo, su consumo por el ser humano puede provocar intoxicaciones e incluso envenenamiento.

Los niveles medios de amigdalina varían de 0,2 a $16 \mathrm{mg} \cdot 100 \mathrm{~g}^{-1}$ en almendras dulces, y entre 3300 y $5400 \mathrm{mg} \cdot 100 \mathrm{~g}^{-1}$ en almendras amargas [1]. Las almendras amargas, por tanto, contienen niveles altos de amigdalina (del 3 al 5\%) y desarrollan el aroma característico del cianuro, por lo que cuantificar la concentración de amigdalina es de gran interés para distinguir entre almendras dulces y amargas. 
Por lo tanto, debido al gran uso que tiene la almendra en la industria alimentaria, tanto en su consumo directo, como en uso como materia prima para la elaboración de productos, es necesario desarrollar métodos que sean capaces de diferenciar entre almendras dulces y amargas.

Uno de los métodos más empleados para la determinación de compuestos cianogénicos en almendras es la cromatografía líquida de alta resolución (HPLC). Diversos autores han realizado estudios con el fin de optimizar el proceso de extracción y estudiar el efecto de la preparación de la muestra para evaluar los niveles de amigdalina y prunasina en almendra $[2,3,4,5,6]$, ya que se considera un método costoso, con tiempos de preparación de muestra largos y la adquisición de instrumentación de alto costo a pesar de los buenos resultados que son obtenidos. Por lo tanto, este trabajo propone optimizar el proceso de extracción y la preparación de la muestra para evaluar los niveles de amigdalina presentes en las almendras.

\section{Materiales y métodos.}

Para este estudio se analizaron un total de 360 almendras, 180 amargas y 180 dulces. Las almendras dulces corresponden a una selección de 30 almendras por cada una de las siguientes variedades: Planeta (P), Comuna (C), Largeta (L), Rumbeta (R), Marcona (M) y Guara (G); mientras que las almendras amargas (A) provienen de distintas variedades sin especificar. Todas las almendras analizadas estaban libres de daños externos o enfermedades, mostrando un color y tamaño uniforme.

Los reactivos y disolventes que se utilizaron fueron amigdalina (BioXtra, $\geq 97.0 \%$ HPLC), acetonitrilo (HPLC Far UV/Gradient Grade), metanol (AGR ACS, ISO, Ph.Eur. Assay $\geq 99,8 \%$ ), acetona (VWR Prolabo) y agua ultrapura que se obtuvo con un desionizador Aquinity (Membrapure $\mathrm{GmbH})$. 
María M. Morales-Padilla; Jacqueline del Rocío Ávila-Jácome; Pascual Barrera-Alina

\section{Condiciones Experimentales}

Para la determinación de amigdalina se empleó un cromatógrafo de líquidos Hitachi LaChrom Elite dotado de una bomba cuaternaria modelo L-2130, un compartimento termostatizado de columnas modelo L-2300 y un detector UV-vis modelo L-2420. La separación se llevó a cabo con una columna Liquid Purple C18 (250 x 4.6mm, 5um) de Análisis Vínicos.

Se utilizó como fase móvil una mezcla binaria de agua ultrapura y acetonitrilo $(80: 20 \mathrm{v} / \mathrm{v})$. La elución se llevó a cabo en modo isocrático con un caudal de $1 \mathrm{~mL}$ min-1, mientras que el volumen de inyección fue de $20 \mu \mathrm{L}$. La detección se llevó a cabo a $218 \mathrm{~nm}$ y para la adquisición de datos se utilizó el software EZChrom v.3.3.2.

Para la extracción de la amigdalina, en primer lugar, se retiró el tegumento de las almendras mediante inmersión en agua caliente durante 5 minutos para después ser secadas a temperatura ambiente. Las almendras se trituraron con un mortero de porcelana de $150 \mathrm{~mL}$, pesado en el triturado obtenido en botes de plástico de $50 \mathrm{~mL}$, en una balanza analítica modelo AB204-S. E1 triturado obtenido de cada una de las almendras se suspendió en $20 \mathrm{~mL}$ de metanol $(>99,8 \%)$ y se mantuvo en agitación constante durante $24 \mathrm{~h}$ empleando un agitador magnético modelo FB15001. Transcurridas las $24 \mathrm{hr}$, la disolución obtenida se filtró a través de un filtro de jeringa de PTFE de 0,22 $\mu \mathrm{m}$. En el caso de las almendras dulces, la disolución filtrada se inyectó directamente en el cromatógrafo, mientras que para las almendras amargas esta disolución se diluyó en una proporción $1: 10(\mathrm{v} / \mathrm{v})$ con metanol.

Con el fin de identificar el pico y cuantificar la amigdalina presente en las almendras, se prepararon dos rectas de calibrado: una para almendras dulces, con un intervalo de concentración 
María M. Morales-Padilla; Jacqueline del Rocío Ávila-Jácome; Pascual Barrera-Alina

comprendido entre $0,1-50 \mathrm{mg} \cdot \mathrm{L}^{-1} ; \mathrm{y}$ un calibrado para las almendras amargas (previamente diluidas), comprendido entre 50 y $1000 \mathrm{mg} \cdot \mathrm{L}^{-1}$.

Optimización de las condiciones de extracción de la amigdalina

Para optimizar las condiciones de extracción de la amigdalina, se analizó tanto el efecto del disolvente como el efecto del tiempo de extracción. En todos los casos, los porcentajes de recuperación se calcularon teniendo en cuenta la cantidad de amigdalina presente inicialmente en la muestra y la cantidad de amigdalina recuperada tras la extracción. Cada experimento se analizó por triplicado.

Para llevar a cabo la optimización, se tomaron 10 almendras dulces (que no contenían amigdalina), se eliminó el tegumento, se trituraron y se fortificaron con un $0,2 \%(\mathrm{~m} / \mathrm{m})$ de patrón de amigdalina. Igualmente, se analizó la eficacia de extracción de 3 disolventes diferentes: metanol, agua y una mezcla metanol: agua 80:20 (v/v). En todos los casos, la extracción se llevó a cabo suspendiendo un $1 \mathrm{~g}$ de almendra triturada conteniendo $0,2 \%(\mathrm{~m} / \mathrm{m})$ de amigdalina con $20 \mathrm{~mL}$ del disolvente, manteniéndolo en agitación constante durante $24 \mathrm{hr}$. Los mejores resultados se obtuvieron empleando metanol como disolvente de extracción con recuperaciones cercanas al 100\% (Figura 1), seleccionándolo para optimizaciones posteriores.

Respecto al proceso de extracción, se probaron diferentes tiempos comprendidos entre 15 min y $32 \mathrm{hr}$, empleando para esto las condiciones experimentales anteriormente mencionadas y 20 $\mathrm{mL}$ de metanol como disolvente. Los resultados obtenidos reflejan una extracción del 100\% después de $24 \mathrm{~h}$ (Figura 2), seleccionando este tiempo para estudios posteriores. 
Optimización de las condiciones de extracción y parámetros cromatográficos en la determinación de amigdalina en las almendras dulces y amargas

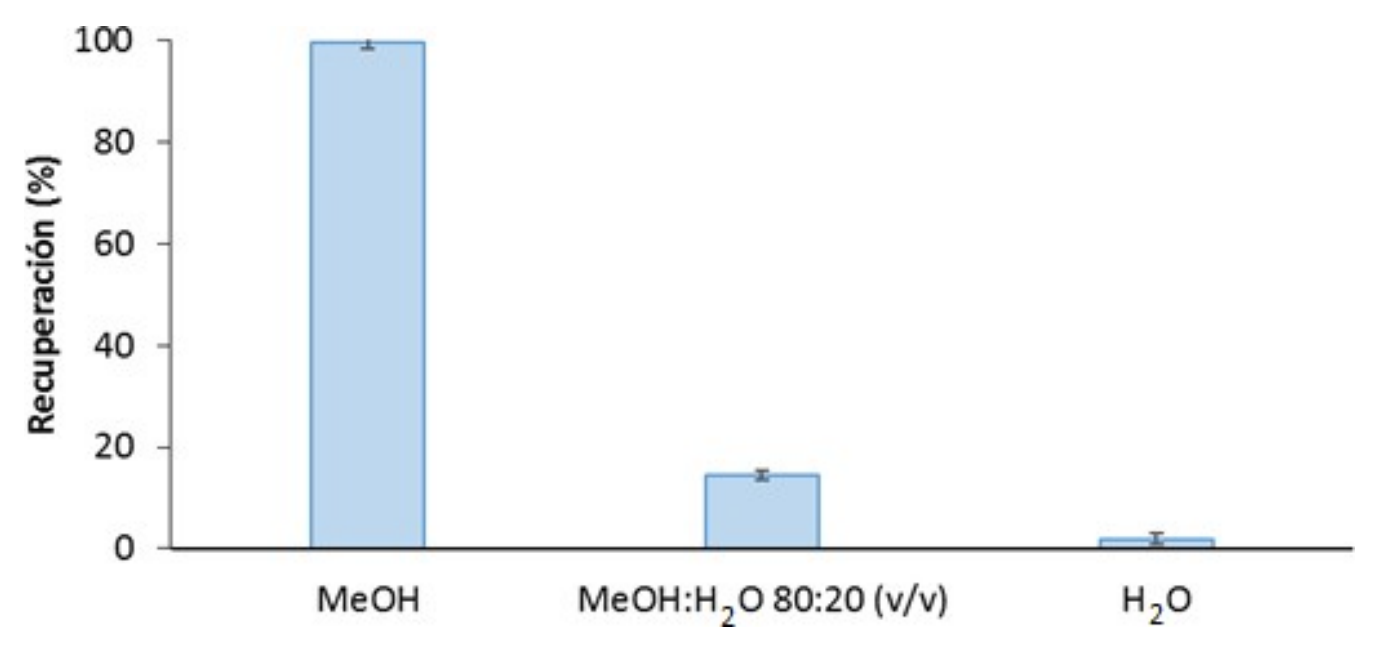

Figura 1. Efecto del disolvente en la recuperación de amigdalina en almendras dulces fortificadas con $0,2 \%(\mathrm{~m} / \mathrm{m})$ de amigdalina.

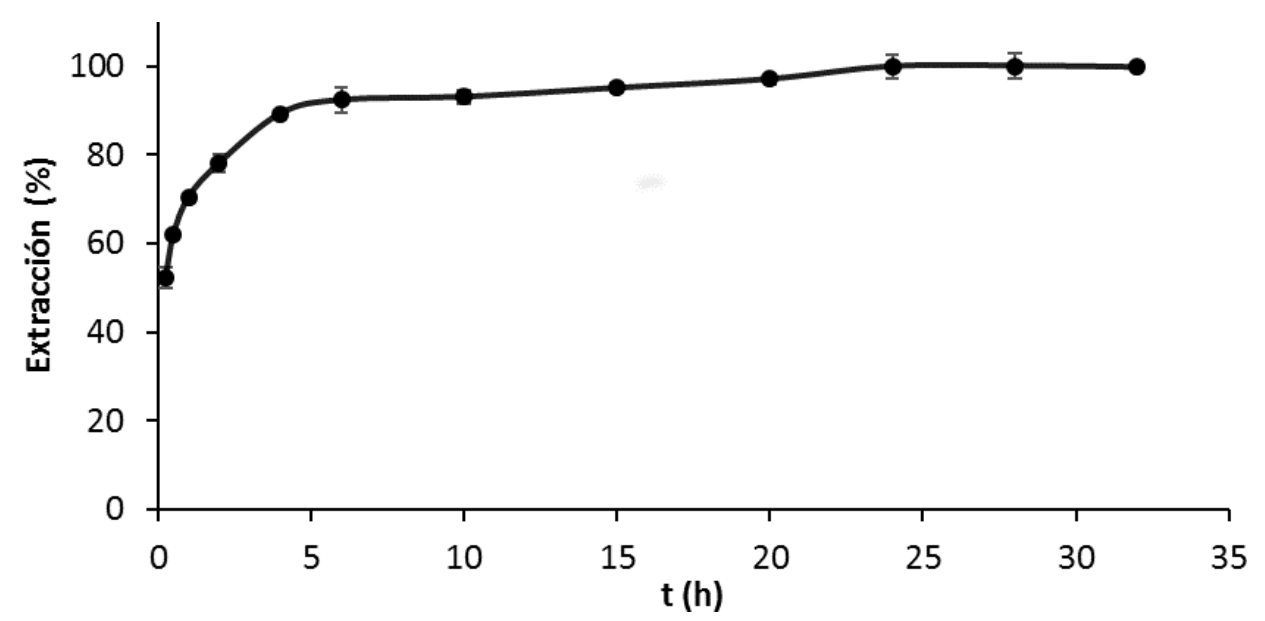

Figura 2. Optimización del tiempo de extracción de amigdalina en almendras dulces fortificadas con $0,2 \%(\mathrm{~m} / \mathrm{m})$ de amigdalina. 


\section{Optimización de los parámetros cromatográficos}

Una vez seleccionadas las condiciones de extracción, se procedió a la optimización de los parámetros cromatográficos, utilizando la misma muestra de almendra fortificada con un $0,2 \%$ $(\mathrm{m} / \mathrm{m})$ de patrón de amigdalina. La optimización se llevó a cabo considerando la mayor resolución del pico de amigdalina respecto a los otros picos procedentes de la matriz que absorben a la máxima longitud de onda de la amigdalina $(218 \mathrm{~nm})$. Dado que la determinación de amigdalina mediante HPLC ha sido llevada a cabo en estudios previos $[2,4,5,6]$, principalmente empleando acetonitrilo $(\mathrm{ACN})$ y agua $\left(\mathrm{H}_{2} \mathrm{O}\right)$ como fase móvil, en este trabajo se analizaron diferentes proporciones de estos disolventes, tanto en elución isocrática como en gradiente, usando un caudal de $1 \mathrm{~mL} \cdot \mathrm{min}^{-1} \mathrm{y}$ un volumen de inyección de $20 \mu \mathrm{L}$. Los mejores resultados en términos de resolución y tiempo de análisis se obtuvieron mediante elución isocrática con $80 \%$ de $\mathrm{H}_{2} \mathrm{O}$ y $20 \% \mathrm{ACN}$, por lo que se seleccionó esta fase móvil para estudios posteriores.

La optimización del caudal fue realizada considerando una variación entre 0,5 y $1,5 \mathrm{~mL} \cdot \mathrm{min}^{-}$

1. Al aumentar el caudal, disminuyó el tiempo de retención, así como la anchura del pico; sin embargo, no se obtuvo una resolución a línea base de un pico de la matriz, así como tampoco, del pico de la amigdalina a caudales superiores a $1,0 \mathrm{~mL} \cdot \mathrm{min}^{-1}$. Por lo tanto, este caudal fue el seleccionado.

Finalmente se estudió el efecto del volumen de inyección, variándose en un intervalo de 5 a $30 \mu \mathrm{L}$. En general, el área del pico de la amigdalina aumentó con el aumento del volumen de inyección. Por lo tanto, se seleccionó $20 \mu \mathrm{L}$ como volumen de inyección en compromiso entre sensibilidad y resolución del pico. 
Parámetros analíticos significativos del método HPLC y su aplicación en la cuantificación de amigdalina en las almendras

La precisión del método desarrollado se determinó mediante el estudio de las repetibilidades intra e inter-día para los tiempos de retención y áreas de pico. Para ello, se inyectó una disolución de amigdalina de $50 \mathrm{mg} \cdot \mathrm{L}^{-1}, 10$ veces por día durante 3 días. Los resultados obtenidos se presentan en la Tabla 1. En todos los casos, los valores de desviación estándar relativa (RSD) obtenidos fueron inferiores a 0,31 y $1,41 \%$ para los tiempos de retención y áreas de pico, respectivamente.

Las curvas de calibración externa se construyeron mediante la inyección de seis disoluciones estándar de amigdalina en los intervalos indicados en la Tabla 1. Como se puede observar, se adoptaron dos intervalos distintos: un intervalo a baja concentración $\left(0,1-50 \mathrm{mg} \cdot \mathrm{L}^{-1}\right)$ que fue utilizado para cuantificar la amigdalina en las almendras dulces y un intervalo a concentraciones más altas $\left(50-1000 \mathrm{mg} \cdot \mathrm{L}^{-1}\right)$, que se utilizó para la cuantificación de la amigdalina en las muestras amargas tras una dilución 1:10 (v/v) de las mismas con metanol. En ambos casos, se obtuvieron líneas rectas con $\mathrm{r}^{2}>0,9996$. Los límites de detección (LOD) y cuantificación (LOQ) fueron calculados para una relación señal/ruido de 3 y 10, respectivamente. Como se observa en la Tabla 1, los valores de LOD y LOQ obtenidos fueron 0,40 y $1,32 \mathrm{mg} \cdot \mathrm{L}^{-1}$, respectivamente. Estos valores fueron ligeramente inferiores a los obtenidos previamente en la literatura $[5,2,3]$.

Además, se prepararon curvas de calibración mediante el método de las adiciones de estándar. Para ello, sobre los extractos de muestra, se adicionaron cuatro disoluciones con concentraciones crecientes de los estándares desde la concentración más baja hasta el límite superior del intervalo empleado (Tabla 1). Las curvas fueron lineales con $\mathrm{r}^{2}>0,9995$; $\mathrm{y}$ en todos los casos, las pendientes de estas curvas de calibrado no difirieron significativamente de las pendientes obtenidas mediante calibración externa. A partir de estos resultados, se puede afirmar que no existe efecto 
María M. Morales-Padilla; Jacqueline del Rocío Ávila-Jácome; Pascual Barrera-Alina

matriz en la determinación de amigdalina en las almendras analizadas. Por lo tanto, se emplearon las curvas de calibración externa para la cuantificación de amigdalina en las muestras.

Tabla 1. Parámetros de calidad del método HPLC desarrollado.

\begin{tabular}{|c|c|c|c|c|c|c|c|c|}
\hline \multirow[t]{2}{*}{ Analito } & \multicolumn{2}{|c|}{$\begin{array}{l}\text { intra-día, } \\
\text { RSD } \\
\mathrm{n}=10 \text { días }\end{array}$} & \multicolumn{2}{|c|}{$\begin{array}{l}\text { inter-día, } \\
\text { RSD } \\
\mathbf{n}=\mathbf{3} \text { días }\end{array}$} & \multirow[t]{2}{*}{$\begin{array}{l}\begin{array}{l}\text { Intervalo } \\
\text { estudiado } \\
\left(\mathrm{mg} \cdot \mathrm{L}^{-1}\right)\end{array} \\
\end{array}$} & \multirow[t]{2}{*}{$r^{2}$} & \multirow[t]{2}{*}{ LOD } & \multirow[t]{2}{*}{ LOC } \\
\hline & $\begin{array}{l}\text { Área } \\
\text { pico }\end{array}$ & $t_{r}$ & $\begin{array}{l}\text { Área } \\
\text { pico }\end{array}$ & $\mathbf{t r}_{\mathbf{r}}$ & & & & \\
\hline Amigdalina & 1,15 & 0,25 & 1,41 & 0,31 & $\begin{array}{l}0,1-50 \\
50-1000\end{array}$ & $\begin{array}{l}0,9996 \\
0,9997\end{array}$ & 0,40 & 1,32 \\
\hline
\end{tabular}

RSD: desviación estándar relativa (\%); tr: tiempo de retención; LOD: límite de detección y LOQ:

límite de cuantificación $\left(\mathrm{mg} \cdot \mathrm{kg}^{-1}\right)$.

La aplicabilidad del método desarrollado se evaluó mediante un estudio de recuperación. Para este propósito, un conjunto de almendras dulces, en las que no se detectó amigdalina, fueron fortificadas con el patrón de amigdalina a 5 niveles de concentración (Tabla 2). Los valores de recuperación que se estimaron, a partir de las cantidades medidas frente a las añadidas, oscilaron entre $98,4 \%$ y $102,9 \%$. Por lo tanto, los resultados obtenidos demostraron la aplicabilidad de la metodología propuesta para la determinación exacta de amigdalina. 
Optimización de las condiciones de extracción y parámetros cromatográficos en la determinación de amigdalina en las almendras dulces y amargas

Tabla 2. Resultados de la recuperación de amigdalina en muestras de almendra en diferentes niveles.

\begin{tabular}{cc}
\hline $\begin{array}{c}\text { Nivel de fortificación } \\
(\boldsymbol{m} / \boldsymbol{m}, \boldsymbol{\%})\end{array}$ & $\begin{array}{c}\text { Recuperación } \\
\mathbf{( \% )}^{\mathbf{a}}\end{array}$ \\
\hline 0,4 & $102,9 \pm 0,9$ \\
0,2 & $99,5 \pm 0,7$ \\
0,1 & $98,4 \pm 1,5$ \\
0,05 & $101,1 \pm 0,8$ \\
0,01 & $100,1 \pm 1,3$ \\
\hline
\end{tabular}

${ }^{\mathrm{a}} \mathrm{Media} \pm \mathrm{RSD}, n=3$

Por otro lado, en la figura 3 se observan los cromatogramas obtenidos, mediante HPLC, del análisis de las almendras, teniendo entonces los gráficos para una almendra amarga (a), una almendra dulce donde se encontró amigdalina (b) y una almendra dulce donde no se detectó la presencia de amigdalina (c).

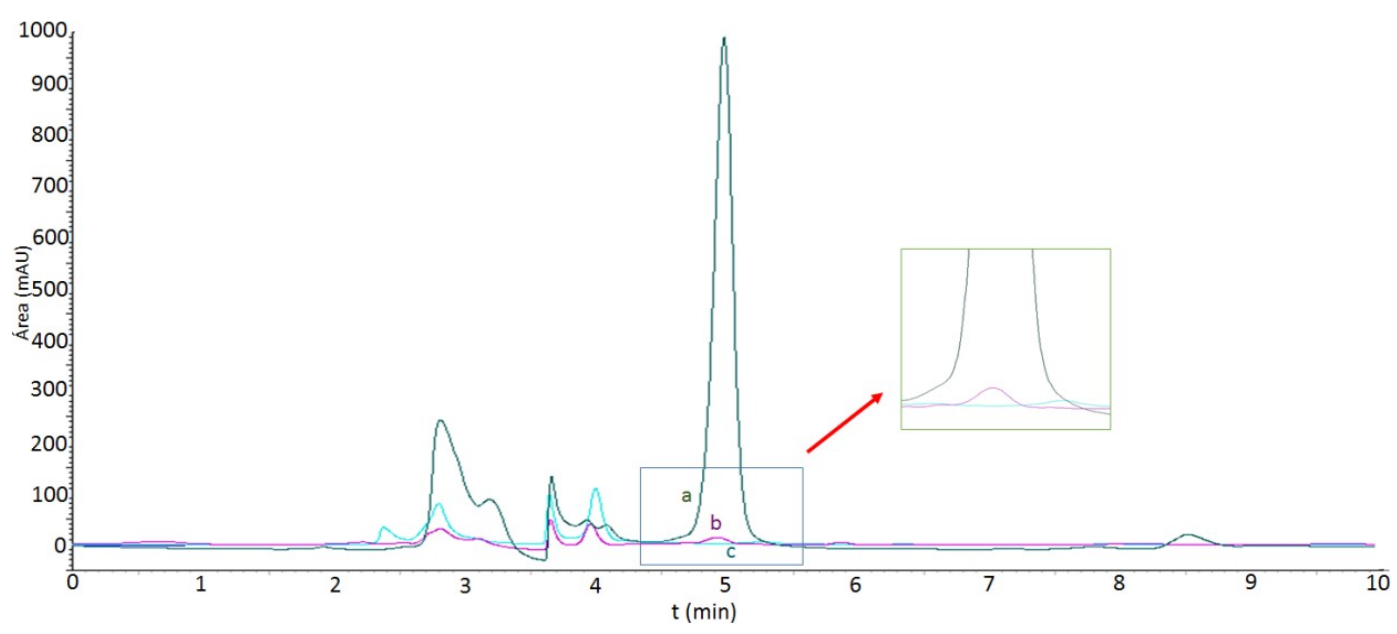

Figura 3. Cromatogramas correspondientes a una almendra a) amarga, b) una dulce con bajo contenido en amigdalina y c) una dulce donde no se detectó amigdalina. 
Una vez inyectadas todas las muestras en el cromatógrafo, se procedió a la cuantificación del contenido de amigdalina en las mismas. En la Tabla 3 se muestran los intervalos de concentración obtenidos para las 180 almendras amargas, y para cada una de las variedades de almendras dulces consideradas. Para el grupo de almendras amargas, el contenido de amigdalina detectado oscila entre 14734 y $50412 \mathrm{mg} \cdot \mathrm{kg}^{-1}$. Estos valores concuerdan con los publicados en la bibliografía [1] con niveles medios de amigdalina para almendras amargas entre 33000 y $54000 \mathrm{mg} \cdot \mathrm{kg}^{-1}$. Por otro lado, el contenido de amigdalina presente en las almendras dulces varía en función de la variedad, registrándose un valor mínimo de $0,67 \mathrm{mg} \cdot \mathrm{kg}^{-1}$ en la variedad Planeta y un valor de $348 \mathrm{mg} \cdot \mathrm{kg}^{-1}$ como valor máximo para la variedad Guara. En la mayoría de los casos, los valores reportados concuerdan con los niveles de amigdalina previamente publicados en la bibliografía (20 - 160 $\left.\mathrm{mg} \cdot \mathrm{kg}^{-1}\right)[1]$

Tabla 3. Intervalos de concentración de amigdalina en almendras amargas y dulces.

\begin{tabular}{|l|l|l|}
\hline Almendras & Variedad & $\begin{array}{l}\text { Contenido de amigdalina } \\
\left(\mathbf{m g} \cdot \mathbf{k g}^{-\mathbf{1}} \mathbf{)}\right.\end{array}$ \\
\hline Amargas & Distintas variedades & $14734,04-50412,56$ \\
\hline \multirow{5}{*}{ Dulces } & Planeta & $0,67-211,27$ \\
\cline { 2 - 3 } & Comuna & $0,96-174,68$ \\
\cline { 2 - 3 } & Largueta & $22,55-184,10$ \\
\cline { 2 - 3 } & Rumbeta & $3,14-18,55$ \\
\cline { 2 - 3 } & Marcona & $6,20-173,19$ \\
\cline { 2 - 3 } & Guara & $52,99-348,13$ \\
\hline
\end{tabular}

\section{Conclusiones.}

Se ha podido desarrollar un método de HPLC capaz de cuantificar la amigdalina presente en almendras dulces y amargas tras la optimización de diversos parámetros experimentales y condiciones cromatográficas. 
Con el método desarrollado se obtuvieron repetibilidades con RSD menores de 0,31 y $1,41 \%$ para los tiempos de retención y áreas de pico, respectivamente. Además, se obtuvo un LOD del 0,40 $\mathrm{mg} \cdot \mathrm{L}^{-1} \mathrm{y}$ recuperaciones en todos los casos cercanas al $100 \%$.

El contenido en amigdalina en almendras dulces varió en un rango de 0,67 a $348 \mathrm{mg} \cdot \mathrm{kg}^{-1}$, en tanto que para las almendras amargas se detectó un contenido de amigdalina entre 14734 y 50412 $\mathrm{mg} \cdot \mathrm{kg}^{-1}$.

Con el desarrollo de este método se podrá cuantificar la amigadalina de una manera eficaz y menos costosa, basándose en la optimización los parámetros experimentales y las condiciones cromatográficas.

\section{Referencias.}

[1] Lee, J., Zhang, G., Wood, E., Rogel Castillo, C., \& Mitchell, A. E. 2013. Quantification of amygdalin in nonbitter, semibitter, and bitter almonds (Prunus dulcis) by UHPLC-(ESI) QqQ MS/MS. Journal of agricultural and food chemistry, 61(32), 7754-7759.

[2] Arrazola, P., Grané, N., Martin, M. L., \& Dicenta, F. 2013. Determination of cyanogenic compound amygdalin and prunasin in almond kernels (prunus dulcis 1) by using liquid chromatography. Revista Colombiana de Química, 42(3), 365-386.

[3] Bolarinwa, I. F., Orfila, C., \& Morgan, M. R. 2014. Amygdalin content of seeds, kernels and food products commercially-available in the UK. Food chemistry, 152, 133-139.

[4] Dicenta, F.; Martinez-Gomez, P.; Grane, N.; Martin, M. L.; Leon, A.; Canovas, J. A.; Berenguer, V. 2002. Relationship between cyanogenic compounds in kernels, leaves, and roots of non-bitter and bitter kernelled almonds. J. Agric. Food Chem., 50(7), 2149-2152.

[5] Ferrara, G., Maggio, P., \& Pizzigallo, M. D. R. 2010. Cyanogenic D-amygdalin contents of the kernels of cultivated almonds and wild Amygdalus webbii Spach. The Journal of Horticultural Science and Biotechnology, 85(5), 410-414.

[6] Yıldırım, A. N., San, B., Koyuncu, F., \& Yıldırım, F. 2010. Variability of phenolics, $\alpha$ tocopherol and amygdalin contents of selected almond (Prunus amygdalus Batsch.) genotypes. J Food Agr Environ, 8, 76-79. 